\title{
BMJ Open Application of three different sets of explicit criteria for assessing inappropriate prescribing in older patients: a nationwide prevalence study of ambulatory care visits in Taiwan
}

Chirn-Bin Chang, ${ }^{1,2}$ Shu-Yu Yang, ${ }^{3,4}$ Hsiu-Yun Lai, ${ }^{5}$ Ru-Shu Wu, ${ }^{4}$ Hsing-Cheng Liu, ${ }^{4,6}$ Hsiu-Ying Hsu, ${ }^{4}$ Shinn-Jang Hwang, ${ }^{7,8}$ Ding-Cheng Chan ${ }^{2,9,10}$

To cite: Chang C-B, Yang S-Y, Lai H-Y, et al. Application of three different sets of explicit criteria for assessing inappropriate prescribing in older patients: a nationwide prevalence study of ambulatory care visits in Taiwan. BMJ Open 2015:5:e08214. doi:10.1136/bmjopen-2015008214

- Prepublication history for this paper is available online To view these files please visit the journal online (http://dx.doi.org/10.1136/ bmjopen-2015-008214).

C-BC and S-YY contributed equally.

Received 16 March 2015 Revised 24 August 2015 Accepted 19 October 2015



For numbered affiliations see end of article.

Correspondence to Dr Ding-Cheng Chan; doctord6226@yahoo.com and Dr Shinn-Jang Hwang; sjhwang@vghtpe.gov.tw

\section{ABSTRACT}

Objective: To investigate the national prevalence of potentially inappropriate medications (PIMs) prescribed in ambulatory care clinics in Taiwan according to three different sets of regional criteria and the correlates of PIM use.

Design: Cross-sectional study.

Setting: This analysis included older patients who visited ambulatory care clinics in 2009 and represented half of the older population included on the Taiwanese National Health Insurance Research Database.

Participants: We identified 1164701 subjects who visited ambulatory care clinics and were over 65 years old in 2009.

Primary and secondary outcome measures: PIM prevalence according to the 2012 Beers criteria, the PIM-Taiwan criteria and the PRISCUS criteria was estimated separately, and characteristics of PIM users were explored. Multivariate logistic regression analysis was used to determine patient factors associated with the use of at least one PIM. Leading PIMs for each set of criteria were also listed.

Results: The prevalence of having at least one PIM at the patient level was highest with the Beers criteria (86.2\%), followed by the PIM-Taiwan criteria $(73.3 \%)$ and the PRISCUS criteria $(66.9 \%)$. Polypharmacy and younger age were associated with PIM use for all three sets of criteria. The leading PIMs detected by the PIM-Taiwan and PRISCUS criteria were all included in the 2012 Beers criteria. Non-COXselective non-steroidal anti-inflammatory drugs in the Beers criteria and benzodiazepines in the PIM-Taiwan and PRISCUS criteria accounted for most leading PIMs.

Conclusions: The prevalence of PIMs was high among older Taiwanese patients receiving ambulatory care visits. The prevalence of PIM and its associated factors varied according to three sets of criteria at the population level.

\section{Strengths and limitations of this study}

- This is a population-based study providing evidence on a high prevalence of potentially inappropriate medication (PIM) use in older Taiwanese patients. Efforts should be made to reduce polypharmacy and PIMs.

- By comparing three sets of PIM criteria, the 2012 Beers criteria are the most comprehensive for PIM identification for older Taiwanese patients. PIM prevalence and associated factors varied among the three sets of criteria at the population level.

- Drug availabilities in Taiwan are different for three sets of PIM criteria. For analysis of associated factors of PIMs, several potential confounders such as detailed information of comorbidities, functional status or living situation were not included because of limitations of the study database.

\section{INTRODUCTION}

The National Health Insurance (NHI) system enrols more than $99 \%$ of residents in Taiwan. ${ }^{1}$ Healthcare services are paid by the National Health Insurance Administration (NHIA) on a fee-for-service basis with a global budget cap. Patients are free to see any healthcare specialty without restrictions and no referral from a primary care physician is needed. ${ }^{2}$ Patients are also free to visit physicians in other residential regions. Older adults use more healthcare resources than younger adults because of more chronic diseases. Moreover, older adults in Taiwan use more healthcare resources than those in other countries. ${ }^{3}$

Drugs are often prescribed to treat chronic diseases in older adults. The pharmacokinetics and pharmacodynamics of these drugs change 
during the aging process and due to chronic disease associated alterations of renal or liver function. Therefore, the incidence of adverse drug events is higher in older adults than in younger adults. These adverse events are important causes of hospitalisation, morbidity and mortality in older adults. ${ }^{4-6}$ Nearly half of these events are considered preventable, $^{7}$ and avoiding prescribing potentially inappropriate medications (PIMs) is an important strategy. Recent studies showed that PIMs were associated with adverse drug reactions (ADRs), ADR-associated medical errors (MEs) and negative clinical outcomes, ${ }^{8}{ }^{9}$ even though the PIMs were not directly responsible for these events. ${ }^{10}$ Explicit criteria of PIMs are defined as a list of drugs which are considered inappropriate in general or for older adults with certain chronic conditions. In contrast, implicit criteria of PIMs are statements that are used to evaluate the appropriateness of individual drugs prescribed for older patients.

Because countries vary in their specific approved drugs and national therapeutic guidelines, many countries have developed their own PIM lists. ${ }^{11}$ The Beers criteria $^{12}$ established in 1991 were the first to be used to reduce prescribing PIMs in nursing homes. The instrument was revised several times for general use among older adults, with the latest version published in $2012 .^{13}$ In the same year, the PIM-Taiwan criteria, which were derived from seven sets of criteria established in different countries, were also published. ${ }^{14}$ In Europe, the German PRISCUS (Latin for 'old and venerable') criteria were shown to discourage PIM use. ${ }^{15-17}$ Many studies based on PIM criteria focus on the prevalence and correlates of PIMs in limited samples, such as inpatient populations or nursing home residents; fewer studies apply these criteria in a general, nationwide population. To the best of our knowledge, few criteria have been used for secondary health insurance claims data analysis studies. ${ }^{17-20} 35$

The aim of this study was to estimate the prevalence of PIMs in a nationwide population prescribed during ambulatory care visits and to investigate the ability of PIM-Taiwan to identify PIMs versus the 2012 Beers criteria and the PRISCUS criteria. We also investigated the factors associated with PIM use and list the leading medications detected by three sets of criteria.

\section{METHODS}

\section{Source of data}

We conducted a secondary data analysis of medical claims from the National Health Insurance Research Database (NHIRD) in Taiwan for 2009. This study was approved by the Research Ethics Committee of National Taiwan University Hospital in 2013. NHIRD collected all claims data of hospitalisation, ambulatory care visits, emergency department visits and home healthcare. In all settings, physicians could prescribe oral, intravenous injected medications or those administered through other routes. Also, physicians could order other therapeutic procedures or programmes, such as surgical interventions or rehabilitation programmes. In this study, only drug prescriptions for the oral route in 2009 covered by NHI were collected for analysis. The patient records and information in this database were anonymised and de-identified before our analysis. We enrolled all patients who were aged 65 years or older who had at least one ambulatory care visit in 2009 if their birthday was an odd number. For confidentiality control, NHI would not release the records for the entire population of older patients for analysis. Thus, this study represents half the population of older Taiwanese patients enrolled in NHI who attended ambulatory care for their acute or chronic illness without considering their place of residence.

\section{Study design and identification of PIM users}

Each brand name oral medication covered by NHI has a unique code. For clarity of medication assignment and to avoid ambiguity, the NHI code was converted to the Anatomical Therapeutic Chemical (ATC) code to efficiently identify PIMs on the database. (This mapping strategy was created by Yea-Huei Kao Yang, Ching-Lan Cheng and Ya-Chuan Chang; their study was called 'To establish therapeutic classification of the pharmaceutical preparations', which was the final report of project no. 102-TFDA-P-044). Information on the pharmacological or chemical subgroups for each individual drug was also available on the WHO Collaborating Center for Drug Statistics Methodology website. ${ }^{21}$ We identified the PIMs independent of chronic conditions because the NHIRD did not contain an exhaustive list of diagnoses for their patients. Therefore, we identified PIMs based on statements that listed PIMs without considering chronic conditions, including table 1 of the original PIM-Taiwan criteria, ${ }^{14}$ the entire PRISCUS criteria ${ }^{22}$ and table 2 of the original 2012 Beers criteria. ${ }^{18}$ Individuals who were prescribed at least one PIM in 2009 based on the three sets of PIM criteria were considered as PIM users. The prevalence and associated factors of PIMs were analysed separately for the three sets of criteria. Age, gender, diagnoses, number of ambulatory care visits, physician visits and hospital visits, and generic names of oral medications were collected for all patients. Also, only three major International Classifications of Diseases, 9th edition Clinical Modification (ICD-9-CM) codes were collected for each ambulatory care visit on the database because of NHI administration specifications. We collected all the diagnoses made during the study period for each participant. The Charlson Comorbidity Index (CCI),${ }^{23}$ which has been validated for higher scores associated with higher risk of death, was calculated based on all diagnoses for each participant with ICD-9-CM codes. Other patient data, such as weight, height, blood pressure, functional status and over-the-counter drug use, were not available due to the limitations of NHIRD.

\section{Statistical analysis}

Patient demographic characteristics (age and gender), comorbidities (CCI) and healthcare resource utilisation 
Table 1 Basic characteristics of Taiwan study population in 2009

\begin{tabular}{|c|c|c|c|c|c|}
\hline $\mathbf{N}(\%)$ & $\begin{array}{l}\text { Overall } \\
1164701(100 \%)\end{array}$ & $\begin{array}{l}65-74 \text { years } \\
639634(54.92 \%)\end{array}$ & $\begin{array}{l}75-84 \text { years } \\
414265(35.57 \%)\end{array}$ & $\begin{array}{l}\geq 85 \text { years } \\
110802(9.51 \%)\end{array}$ & p Value \\
\hline Gender & & & & & $<0.01$ \\
\hline Male & 558904 (47.99) & $296436(46.34)$ & $210250(50.75)$ & $52218(47.13)$ & \\
\hline Female & 605797 (52.01) & 343198 (53.66) & 204015 (49.25) & $58584(52.87)$ & \\
\hline Number of diagnoses in 1 year & & & & & $<0.01$ \\
\hline $1-10$ & 633846 (54.42) & 362956 (56.74) & $209045(50.46)$ & $61845(55.82)$ & \\
\hline$>10$ & $530816(45.58)$ & 276657 (43.26) & 205207 (49.54) & $48952(44.18)$ & \\
\hline Charlson Comorbidity Index & & & & & $<0.01$ \\
\hline Score 1 & 268123 (23.02) & $148125(23.16)$ & 94506 (22.81) & $25492(23.01)$ & \\
\hline Score 2 & 183247 (15.73) & $94274(14.74)$ & 69395 (16.75) & $19578(17.67)$ & \\
\hline Score $>2$ & 299585 (25.72) & 140397 (21.95) & 124567 (30.07) & 34621 (31.25) & \\
\hline $\begin{array}{l}\text { Number of outpatient clinic visits in } \\
1 \text { year }\end{array}$ & & & & & $<0.01$ \\
\hline $1-24$ & $656878(56.40)$ & 383403 (59.94) & $210536(50.82)$ & $62939(56.80)$ & \\
\hline$>24$ & $507823(43.60)$ & 256231 (40.06) & 203729 (49.18) & $47863(43.20)$ & \\
\hline Number of hospital visits in 1 year & & & & & $<0.01$ \\
\hline $1-4$ & $783351(67.26)$ & $419052(65.51)$ & $279393(67.44)$ & 84906 (76.63) & \\
\hline$>4$ & $381350(32.74)$ & $220582(34.49)$ & $134872(32.56)$ & 25896 (23.37) & \\
\hline Number of medications in 1 year & & & & & $<0.01$ \\
\hline $1-20$ & $659452(56.62)$ & $375455(58.70)$ & $218320(52.70)$ & $65677(59.27)$ & \\
\hline$>20$ & 505249 (43.38) & 264179 (41.30) & $195945(47.30)$ & $45125(40.73)$ & \\
\hline
\end{tabular}

(frequency of clinic visits, number of hospital visits and number of oral medications prescribed) for 2009 were investigated for their association with PIM use. CCI was stratified as a score of 1, 2 or more than 2. We used stepwise multivariate logistic regression models to identify the correlates of having at least one PIM at the patient level. Each set of criteria was analysed separately to determine its associated factors. The statistical significance $\alpha$ was set at $\mathrm{p}<0.05$. We also ranked the leading PIMs from each set of PIM criteria based on PIMs accounting for more than $1 \%$ of total prescriptions. Data were analysed using SAS for Windows V.9.2 (SAS Institute Inc, Cary, North Carolina, USA).

\section{RESULTS}

Basic characteristics of study subjects

As shown in table 1, a total of 1164701 older patients were enrolled in this study. More than half $(55 \%)$ were aged $65-74$ years and $48 \%$ were men. Nearly half of the patients had more than 10 diagnoses and nearly $40 \%$ had a score of at least 2 on the CCI, which indicates a high disease burden for our study population. The group of patients aged over 85 years had the highest proportion (49\%) scoring at least 2 on the CCI among three age stratifications. Healthcare resource use was also high: $43 \%$ of patients exceeded 24 ambulatory care visits and $32 \%$ of patients visited more than four hospitals in 1 year. Among three age groups, patients aged 75-84 years had the highest rate of clinic visits, but those aged 6574 years visited the most hospitals. All patients in our study population had been prescribed at least one drug during their ambulatory clinic visits. Forty-three percent of all patients were prescribed more than 20 different medications in 1 year, especially those aged $74-85$ years. Those who were aged over 85 years had the lowest rates of hospital visits and the lowest number of medications.

\section{Description of PIM users}

The characteristics of three sets of PIM criteria are listed in table 2. The 2012 Beers criteria included the highest

Table 2 Characteristics of the three sets of explicit criteria and their performance in detecting potentially inappropriate medications (PIMs) in Taiwanese study patients in 2009

\begin{tabular}{llll}
\hline & Beers criteria & PIM-Taiwan criteria & PRISCUS criteria \\
\hline Year of criteria & 2012 & 2012 & 2010 \\
Country & USA & Taiwan & German \\
Statements* & 34 & 24 & 15 \\
Number of medicationst & 137 & 84 & 83 \\
Availability of PIMs listed in each set of criteria in Taiwan & $105(76.64 \%)$ & $84(100 \%)$ & $69(84.15 \%)$ \\
Number of patients with any PIMs & $1004234(86.22 \%)$ & $853915(73.32 \%)$ & $778825(66.87 \%)$ \\
\hline
\end{tabular}

*Statements are those for potentially inappropriate medications (PIMs) without considering drug-disease or drug-syndrome interactions. †Medications are considered as potentially inappropriate without considering drug-disease or drug-syndrome interactions. 
number of statements and individual drugs, and the numbers of individual drugs in the PIM-Taiwan and PRISCUS criteria were only about $60 \%$ of the Beers' lists. The availability of PIMs is highest in Taiwan but lowest in the 2012 Beers criteria (100\% vs 76\%). After considering medication availability, the 2012 Beers criteria still had the highest number of individual drugs in three sets of criteria. The prevalence of patients receiving at least one PIM during the year was $86 \%, 73 \%$ and $67 \%$ using the Beers criteria, the PIM-Taiwan criteria and the PRISCUS criteria, respectively.

By applying the 2012 Beers criteria, 87\% of women had been prescribed at least one PIM (table 3). Among the patients receiving PIMs from the three sets of criteria, those aged 74-85 years had the highest risk of being prescribed at least one PIM using the 2012 Beers criteria and the PRISCUS criteria. However, those aged 65-74 years had the highest risk of being prescribed medications listed in the PIM-Taiwan criteria. Patients having a higher number of diagnoses were at high risk of being prescribed PIMs compared with those with a lower number of diagnoses. In accordance with their high disease burden, when patient visits exceeded 24 ambulatory clinics and more than four hospitals, they had a higher risk of receiving PIMs. The percentage of patients having PIMs listed in the 2012 Beers criteria increased to over $95 \%$ if patients exceeded 24 clinic visits, four different hospital visits and 20 different medications.

\section{Association factors of PIMs}

In multivariate analysis, male patients were more likely than female patients to be prescribed PIMs listed in the Beers and PRISCUS criteria (table 4). Patients aged over 75 (75-85, and 85 and older) were more likely to receive PIMs listed in the PRISCUS criteria only. Patients who were prescribed more medications in 2009 were more likely to have PIMs across all three sets of criteria. When a patient was prescribed one more drug, the odds of having PIMs increased the most $(\mathrm{OR}=1.6)$ when the Beers criteria were used for assessment. Inverse relationships were found between CCI score and the use of at least one PIM, except when using the Beers criteria.

\section{Leading medications in three sets of PIM criteria}

Overall, 27485169 prescriptions were written for our study population in 2009. The leading PIMs varied in the three sets of criteria. Twenty-three medications had a prevalence higher than $1 \%$ of total prescriptions. Nineteen medications were identified by the Beers criteria (table 5), while eight were identified by the PIM-Taiwan criteria and nine by the PRISCUS criteria. In the Beers and PRISCUS criteria, psychotropic drugs and non-steroidal anti-inflammatory drugs (NSAIDs) accounted for the most frequent PIMs. In contrast, the most frequent PIMs according to the PIM-Taiwan criteria were first-generation histamine H1-receptor antagonists. Diclofenac accounted for 5\% of total study population prescriptions and was ranked first by the Beers criteria.

\begin{tabular}{|c|c|c|c|c|c|c|}
\hline $\mathbf{N}(\%)$ & 2012 Beers & p Value & PIM-Taiwan & p Value & PRISCUS & p Value \\
\hline Patient number & $1004243(86.22)$ & & 853915 (73.32) & & 778825 (66.87) & \\
\hline Gender & & $<0.01$ & & $<0.01$ & & $<0.01$ \\
\hline Male & 476549 (85.26) & & $397196(71.07)$ & & 368560 (65.94) & \\
\hline Female & 527694 (87.11) & & 456719 (75.39) & & 410265 (67.72) & \\
\hline Age (years) & & $<0.01$ & & $<0.01$ & & $<0.01$ \\
\hline $65-74$ & 548368 (85.73) & & $473753(74.07)$ & & 407054 (63.64) & \\
\hline $75-84$ & 361763 (87.33) & & 305697 (73.79) & & $294762(71.15)$ & \\
\hline$\geq 85$ & $94112(84.94)$ & & 74465 (67.21) & & 77009 (69.50) & \\
\hline Number of diagnoses & & $<0.01$ & & $<0.01$ & & $<0.01$ \\
\hline $1-10$ & $486807(76.80)$ & & 372386 (58.75) & & 326566 (51.52) & \\
\hline$>10$ & 517433 (97.48) & & 481526 (90.71) & & $452258(85.20)$ & \\
\hline Charlson Comorbidity Index & & $<0.01$ & & $<0.01$ & & $<0.01$ \\
\hline Score 1 & 232362 (86.66) & & $195198(72.80)$ & & $181090(67.54)$ & \\
\hline Score 2 & 162592 (88.73) & & $137535(75.05)$ & & $132979(72.57)$ & \\
\hline Score $>2$ & 279879 (93.42) & & 241659 (80.66) & & 242536 (80.96) & \\
\hline $\begin{array}{l}\text { Number of outpatient clinic visits in } \\
1 \text { year }\end{array}$ & & $<0.01$ & & $<0.01$ & & $<0.01$ \\
\hline $1-24$ & $514117(78.27)$ & & 406031 (61.81) & & $349443(53.20)$ & \\
\hline$>24$ & 490126 (96.52) & & 447884 (88.20) & & $429382(84.55)$ & \\
\hline Number of hospital visits in 1 year & & $<0.01$ & & $<0.01$ & & $<0.01$ \\
\hline $1-4$ & 532047 (76.57) & & $501371(64.00)$ & & 457348 (58.38) & \\
\hline$>4$ & $372196(98.82)$ & & 352544 (92.45) & & 321477 (84.3) & \\
\hline Number of medications in 1 year & & $<0.01$ & & $<0.01$ & & $<0.01$ \\
\hline $1-20$ & 504939 (76.57) & & 379265 (57.51) & & $336536(51.03)$ & \\
\hline$>20$ & 499304 (98.82) & & 474650 (93.94) & & 442289 (87.54) & \\
\hline
\end{tabular}


Table 4 Multivariate logistic regression analysis for having at least one drug as potentially inappropriate $(\mathrm{N}=1164701)$

\begin{tabular}{|c|c|c|c|}
\hline & \multicolumn{3}{|l|}{ OR (95\% Cl) } \\
\hline & 2012 Beers & PIM-Taiwan & PRISCUS \\
\hline \multicolumn{4}{|l|}{ Gender } \\
\hline Male & 1 & 1 & 1 \\
\hline Female & $0.93(0.93 \text { to } 0.93)^{*}$ & $1.02(1.02 \text { to } 1.02)^{*}$ & $0.87(0.87 \text { to } 0.88)^{\star}$ \\
\hline \multicolumn{4}{|l|}{ Age (years) } \\
\hline $65-74$ & 1 & 1 & 1 \\
\hline $75-84$ & $0.94(0.94 \text { to } 0.94)^{\star}$ & $0.93(0.93 \text { to } 0.93)^{*}$ & $1.05(1.04 \text { to } 1.05)^{\star}$ \\
\hline$\geq 85$ & $0.89(0.89 \text { to } 0.90)^{*}$ & $0.82(0.82 \text { to } 0.83)^{*}$ & $1.03(1.02 \text { to } 1.03)^{*}$ \\
\hline \multicolumn{4}{|l|}{ Charlson Comorbidity Index } \\
\hline Score 1 & 1 & 1 & 1 \\
\hline Score 2 & $0.88(0.88 \text { to } 0.88)^{*}$ & $0.85(0.85 \text { to } 0.85)^{*}$ & $0.89(0.88 \text { to } 0.89)^{*}$ \\
\hline Score $>2$ & $1.03(1.03 \text { to } 1.04)^{*}$ & $0.89(0.89 \text { to } 0.91)^{*}$ & $0.90(0.90 \text { to } 0.91)^{*}$ \\
\hline Number of medications $\dagger$ & $1.60(1.60 \text { to } 1.60)^{*}$ & $1.56(1.56 \text { to } 1.56)^{\star}$ & $1.46(1.46 \text { to } 1.46)^{\star}$ \\
\hline
\end{tabular}

\section{DISCUSSION}

This is the first nationally representative evaluation of PIM use with an instrument developed in Taiwan. We were able to compare the results of the PIM-Taiwan criteria with the results of two other sets of well established PIM criteria. All three sets of PIM criteria identified high percentages of older adults who had been prescribed at least one PIM in the ambulatory care setting. PIM prevalence estimated from the PIM-Taiwan criteria ranked in the middle among the three sets of criteria.
Generally, polypharmacy and being aged 65-74 years were associated with more PIM use.

The annual prevalence of PIMs in our population is higher than that reported in previous community-based or health insurance studies (Beers range 28-65\% and PRISCUS 22-25\%). ${ }^{17} 24-26$ Only one study applying the Beers criteria in an Indian hospitalised population showed a high rate of PIM exposure similar to Taiwan's. Several factors are responsible for the relatively high prevalence in our study. First, the 2012 version of the

Table 5 The leading PIMs* identified in 27485169 prescriptions in a Taiwanese study population in 2009

\begin{tabular}{|c|c|c|c|}
\hline Drug name and anatomical (ATC) code/prevalence of exposure (\%) & 2012 Beers & PIM-Taiwan & PRISCUS \\
\hline 1 Diclofenac (M01AB05)/5.07\% & $\checkmark$ & & \\
\hline 2 Cimentidine (A02BA01)/3.69\% & & $\checkmark$ & \\
\hline 3 Clorzoxazone (M03BВ03)/2.88\% & $\checkmark$ & $\checkmark$ & \\
\hline 4 Zolpidem (N05CF02)/2.80\% & 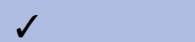 & & $\checkmark$ \\
\hline 5 Alprazolam (N05BA12)/2.51\% & $\checkmark$ & & $\checkmark$ \\
\hline 6 Dipyridamole (B01AC07)/2.45\% & $\checkmark$ & & \\
\hline 7 Mefenamic acid (M01AG01)/2.30\% & $\checkmark$ & & \\
\hline 8 Lorazepam (N05BA06)/2.08\% & $\checkmark$ & & $\checkmark$ \\
\hline 9 Nifedipine $(\mathrm{B} 01 \mathrm{AC} 05) / 2.01 \%$ & $\checkmark$ & & $\checkmark$ \\
\hline 10 Ibuprofen (M01AE01)/1.90\% & 2 & & \\
\hline 11 Metoclopramide (A03FA01)/1.76\% & $\checkmark$ & & \\
\hline 12 Doxazosin (C02CA04)/1.74\% & 2 & & $\checkmark$ \\
\hline 13 Meloxicam (M01AC06)/1.56\% & $\checkmark$ & & $\checkmark$ \\
\hline 14 Dexchlorpheniramine (R06AB02)/1.55\% & $\checkmark$ & $\checkmark$ & \\
\hline 15 Pirecetam (N06BX03)/1.46\% & & & $\checkmark$ \\
\hline 16 Mequitazine (R06AD07)/1.43\% & & $\checkmark$ & \\
\hline 17 Diazepam (N05BA01)/1.31\% & $\checkmark$ & $\checkmark$ & $\checkmark$ \\
\hline 18 Clonazepam (N03AE01)/1.28\% & $\checkmark$ & $\checkmark$ & \\
\hline 19 Chlorpheniramine (R06AB54)/1.24\% & $\checkmark$ & $\checkmark$ & \\
\hline 20 Terazosin (G04CA03)/1.18\% & $\checkmark$ & & $\checkmark$ \\
\hline 21 Estazolam (N05CD04)/1.17\% & $\checkmark$ & & \\
\hline 22 Fludiazepam (N05BA17)/1.10\% & & $\checkmark$ & \\
\hline 23 Glibenclamide (A10BB01)/1.07\% & $\checkmark$ & & \\
\hline
\end{tabular}


Beers criteria added more medications as PIMs, including all non-COX-selective NSAIDs and benzodiazepine regardless of their half-life. Second, under the NHIA policy to control the growth of drug expenditure, physicians may prefer to prescribe cheaper drugs. Therefore, cheaper drugs including generic first antihistamine and non COX-II selective NSAIDs accounted for the high prevalence of PIMs in Taiwan. ${ }^{27}$ Third, our NHI offered unrestricted access to physicians and almost all prescription medications. ${ }^{2}$ Therefore, Taiwanese patients made more frequent visits to ambulatory care clinics than older American patients ${ }^{28}$ and polypharmacy is highly prevalent in Taiwan compared with western countries, ${ }^{18}$ 29-31 which certainly increases the risk of PIM use. $^{25} 32$ There were only limited studies of polypharmacy prevalence in Asia but this problem was also apparent in Korea. ${ }^{33}$ Although the PIM-Taiwan criteria were established for local use in Taiwan, most individual PIMs in our criteria were included in the Beers criteria and other country-specific PIM criteria. It is convenient for other regions without their own PIM criteria to apply this tool to measure prescription quality at the population level. Nevertheless, PIM prevalence in Taiwan only represented local prescription preference and health resource utilisation under the coverage of the NHI of Taiwan. Also, the availabilities of drugs listed in the 2012 Beers criteria and the PRISCUS criteria were different, which suggests that the drug markets and prescription patterns are different in the USA, Germany and Taiwan. Our findings would be applicable to healthcare systems with universal health insurance and relatively unrestricted medication prescriptions.

Education of physicians, pharmacists and patients about PIM lists will increase awareness and possibly decrease PIM use. ${ }^{34}$ Also, computer-assisted prescription alert programmes incorporated in PIM lists would provide possible therapeutic alternatives, such as the PIM-Taiwan and PRISCUS criteria, and may help to reduce use of these high-risk medications. ${ }^{36}$ In the near future, a computer assisted prescription warning system will be incorporated into our NHI identification card based on table 1 of the PIM-Taiwan criteria. Physicians will be discouraged from prescribing these medications for older adults at each clinical encounter. Lastly, including fewer PIMs in a hospital pharmacy, for example, purchase famotidine rather than cimentidine, is also an effective strategy to reduce PIM use.

For factors associated with PIMs, we found different patterns of risk between three sets of PIM criteria. Several studies applying the 2012 Beers and PRISCUS criteria found that female gender was associated with use of more PIMs. ${ }^{37}$ Various clinical settings and study populations can account for these differences. In our study population, acute upper respiratory infections, benign prostate hypertrophy and contact dermatitis were leading diagnoses and also more prevalent in our male patients (data not shown). The 2012 Beers criteria and PRISCUS criteria included more individual drugs such as $\alpha-1$ blockers and first-generation antihistamines; therefore, the risk of being prescribed PIMs was increased in male patients. For the PIM-Taiwan criteria, the literature was limited. ${ }^{11}$ Contrary to our previous study on home care service recipients, female patients were more likely to be prescribed PIMs using the PIM-Taiwan criteria in the current study. A possible explanation is that cimentidine, muscle relaxants and short-acting benzodiazepines were major PIMs in the PIM-Taiwan criteria. The prevalence of functional digestive disorders, disease of the musculoskeletal system and mental disorders was higher among women; therefore, their risk of receiving PIMs increased. As for the association between the CCI and PIM use, it is difficult to reach a conclusion. In previous studies, positive and negative associations between the CCI and PIMs were observed. ${ }^{9} 3940$ We found that older patients with a CCI score of 1 had the highest risk when we applied the PIM-Taiwan and PRISCUS criteria. However, on applying the Beers criteria, patients with a CCI of 3 had the highest risk. The only explanation is a cautious prescribing attitude of physicians to reduce prescribing medications that have a higher risk of side effects for older patients with multiple comorbidities. ${ }^{40}$ Our database only included patients' diseases which can be classified using ICD-9-CM codes; therefore, the results should be interpreted with caution because other important risk factors for PIM use, such as functional status, were not included in the analysis. Further prospective studies investigating the attitude of physicians towards PIMs are necessary.

NSAIDs, benzodiazepines and first-generation antihistamines were major offending PIMs in this study, and these results are consistent with previous studies using the 2012 Beers and PRISCUS criteria. ${ }^{3} 11 \quad 14 \quad 1726 \quad 39$ Almost all PIMs identified by the PIM-Taiwan and PRISCUS criteria are included in the 2012 Beers criteria. Only certain non-COX-selective NSAIDs and long-acting benzodiazepines are listed in the PIM-Taiwan criteria, and similarly in the PRISCUS criteria. In contrast, the 2012 Beers criteria include all non-COX-selective NSAIDs and long, intermediate and short-acting benzodiazepines. This comprehensive inclusion of benzodiazepines was supported by several studies. ${ }^{41}{ }^{42}$ Almost all PIM criteria were established based on expert consensus; therefore, they should be used to support the prescribing decision not to substitute the physician's decision on prescription. Although some studies show that drugs in the PIM criteria contributed to only a small percentage of ADRs, ${ }^{43}$ a recent study with a different design demonstrated an association between PRISCUS drugs and ADRs. ${ }^{10}$ More importantly, Koyama and colleagues found that patients taking PIMs and drugs with high anticholinergic effects are at higher risk of functional and cognitive impairment in a 5-year prospective cohort study. ${ }^{44}$ Their study implied that the adverse events related to PIMs may be difficult to identify in crosssectional studies. Further study is needed to confirm the 
impact of these PIM lists on the quality of life, healthcare resource use and mortality of older adults. ${ }^{45}$

This study has several strengths. First, it was a population-based study including half of the total population of older patients included on the Taiwanese NHIRD. Therefore, the data are highly representative. Limited studies have included national population data to evaluate PIM criteria. Second, we selected the evidence-based Beer criteria and well studied PRISCUS criteria for comparison with the PIM-Taiwan criteria. Although the 2012 Beers criteria seem to be more comprehensive, the PIM-Taiwan criteria which only contains half the number of individual drugs as the 2012 Beer criteria can identify $80 \%$ of PIMs prescribed for older Taiwanese patients. PIM-associated factors also varied among the three sets of criteria. This suggests that region-specific criteria are better tools for evaluating prescription quality in specific drug markets and health insurance systems. Third, all prescribed medications are covered by the NHI, which means our database contains detailed information of medications, including combination drugs.

Our study is a secondary data analysis with fundamental limits. First, only three diagnoses were made for one ambulatory care visit. Therefore, we only adapted PIM criteria independent of chronic diseases. Second, some drugs listed in the Beers criteria (meprobamate, trimethobenzamide, oxaprozin etc.) and the PRISCUS criteria (e.g. metildigoxin, tetrazepam, briotizolam etc.) are not available in Taiwan, but all drugs in the PIM-Taiwan criteria are available. Drug availability is one of the major determinants of PIM prevalence. ${ }^{32}$ Even so, we still found that the Beers criteria detected more PIMs than the other criteria, mainly due to the inclusion of more medications on the list. Third, to analyse associated factors of PIMs, not all potential confounders such as detailed information of comorbidities, functional status or living situation were included because of limitations of the study database.

In conclusion, the prevalence of PIM users in our population is over $65 \%$. The prevalence was highest for the 2012 Beers criteria, which also included most leading PIMs in the PIM-Taiwan and PRISCUS criteria. The prevalence of PIMs and associated factors varied among the three sets of criteria at the population level.

\author{
Author affiliations \\ ${ }^{1}$ Division of Internal Medicine, National Taiwan University Chu-Tung Branch, \\ Hsinchu County, Taiwan \\ ${ }^{2}$ Department of Geriatrics and Gerontology, National Taiwan University \\ Hospital, Taipei, Taiwan \\ ${ }^{3}$ College of Pharmacy, Kaohsiung Medical University, Kaohsiung, Taiwan \\ ${ }^{4}$ Taipei City Psychiatry Center, Taipei City Hospital, Taipei, Taiwan \\ ${ }^{5}$ Department of Family Medicine, National Taiwan University Hospital Hsin- \\ Chu Branch, Hsinchu City, Taiwan \\ ${ }^{6}$ Department of Psychiatry, School of Medicine, Taipei Medical University, \\ Taipei, Taiwan \\ ${ }^{7}$ Department of Family Medicine, Taipei Veteran General Hospital, Taipei, \\ Taiwan \\ ${ }^{8}$ National Yang-Ming University, School of Medicine, Taipei, Taiwan
}

${ }^{9}$ Department of Internal Medicine, National Taiwan University Hospital, Taipei, Taiwan

${ }^{10}$ Superintedent Office, National Taiwan University Hospital Chu-Tung Branch, Hsinchu County, Taiwan

Contributors C-BC, S-JH, D-CC, H-YL wrote and designed the study. S-YY, R-SW, H-CL and H-YH designed the study and analysed the data.

Funding This research is funded by Taipei City Government (10101-62-025). Competing interests None declared.

Ethics approval Research Ethics Committee of National Taiwan University Hospital.

Provenance and peer review Not commissioned; externally peer reviewed.

Data sharing statement We did have additional unpublished data from the study.

Open Access This is an Open Access article distributed in accordance with the Creative Commons Attribution Non Commercial (CC BY-NC 4.0) license, which permits others to distribute, remix, adapt, build upon this work noncommercially, and license their derivative works on different terms, provided the original work is properly cited and the use is non-commercial. See: http:// creativecommons.org/licenses/by-nc/4.0/

\section{REFERENCES}

1. Cahir C, Moriarty F, Teljeur C, et al. Potentially inappropriate prescribing and vulnerability and hospitalization in older community-dwelling patients. Ann Pharmacother 2014;48: 1546-54.

2. Cheng TM. Taiwan's new national health insurance program: genesis and experience so far. Health Aff (Millwood) 2003;22:61-76.

3. Jhaveri BN, Patel TK, Barvaliya MJ, et al. Utilization of potentially inappropriate medications in elderly patients in a tertiary care teaching hospital in India. Perspect Clin Res 2014;5:184-9.

4. Onder G, Pedone C, Landi F, et al. Adverse drug reactions as cause of hospital admissions: results from the Italian Group of Pharmacoepidemiology in the Elderly (GIFA). J Am Geriatr Soc 2002;50:1962-8.

5. Jano E, Aparasu RR. Healthcare outcomes associated with Beers' criteria: a systematic review. Ann Pharmacother 2007:41:438-47.

6. Perri M III, Menon AM, Deshpande AD, et al. Adverse outcomes associated with inappropriate drug use in nursing homes. Ann Pharmacother 2005;39:405-11.

7. Hakkarainen KM, Hedna K, Petzold M, et al. Percentage of patients with preventable adverse drug reactions and preventability of adverse drug reactions - a meta-analysis. PLoS ONE 2012;7: e33236.

8. Lu WH, Wen YW, Chen LK, et al. Effect of polypharmacy, potentially inappropriate medications and anticholinergic burden on clinical outcomes: a retrospective cohort study. CMAJ 2015;187:E130-7.

9. Montastruc F, Duguet C, Rousseau V, et al. Potentially inappropriate medications and adverse drug reactions in the elderly: a study in a PharmacoVigilance database. Eur J Clin Pharmacol 2014;70: 1123-7.

10. Dormann $\mathrm{H}$, Sonst $\mathrm{A}$, Muller $\mathrm{F}$, et al. Adverse drug events in older patients admitted as an emergency: the role of potentially inappropriate medication in elderly people (PRISCUS). Dtsch Arztebl Int 2013;110:213-19.

11. Chang CB, Lai HY, Yang SY, et al. Patient- and clinic visit-related factors associated with potentially inappropriate medication use among older home healthcare service recipients. PLOS ONE 2014;9: e94350.

12. Beers MH, Ouslander JG, Rollingher I, et al. Explicit criteria for determining inappropriate medication use in nursing home residents. UCLA Division of Geriatric Medicine. Arch Intern Med 1991;151: 1825-32.

13. American Geriatrics Society 2012 Beers Criteria Update Expert Panel. American Geriatrics Society updated Beers Criteria for potentially inappropriate medication use in older adults. J Am Geriatr Soc 2012;60:616-31

14. Chang CB, Yang SY, Lai HY, et al. Using published criteria to develop a list of potentially inappropriate medications for elderly patients in Taiwan. Pharmacoepidemiol Drug Saf 2012;21:1269-79.

15. Holt S, Schmiedl S, Thurmann PA. Potentially inappropriate medications in the elderly: the PRISCUS list. Dtsch Arztebl Int 2010;107:543-51. 
16. Fromm MF, Maas R, Tumena T, et al. Potentially inappropriate medications in a large cohort of patients in geriatric units: association with clinical and functional characteristics. Eur J Clin Pharmacol 2013;69:975-84

17. Schubert I, Kupper-Nybelen J, Ihle P, et al. Prescribing potentially inappropriate medication (PIM) in Germany's elderly as indicated by the PRISCUS list. An analysis based on regional claims data. Pharmacoepidemiol Drug Saf 2013;22:719-27.

18. Blozik E, Rapold R, von Overbeck J, et al. Polypharmacy and potentially inappropriate medication in the adult, community-dwelling population in Switzerland. Drugs Aging 2013;30:561-8.

19. Lane CJ, Bronskill SE, Sykora K, et al. Potentially inappropriate prescribing in Ontario community-dwelling older adults and nursing home residents. J Am Geriatr Soc 2004;52:861-6.

20. Mann E, Haastert B, Frühwald $T$, et al. Potentially inappropriate medication in older persons in Austria: a nationwide prevalence study. Eur Geriatr Med 5:399-405.

21. Saarelainen LK, Turner JP, Shakib S, et al. Potentially inappropriate medication use in older people with cancer: prevalence and correlates. J Geriatr Oncol 2014;5:439-46.

22. Goltz L, Kullak-Ublick GA, Kirch W. Potentially inappropriate prescribing for elderly outpatients in Germany: a retrospective claims data analysis. Int J Clin Pharmacol Ther 2012;50:185-94.

23. Deyo RA, Cherkin DC, Ciol MA. Adapting a clinical comorbidity index for use with ICD-9-CM administrative databases. J Clin Epidemiol 1992;45:613-19.

24. Amann U, Schmedt N, Garbe E. Prescribing of potentially inappropriate medications for the elderly: an analysis based on the PRISCUS list. Dtsch Arztebl Int 2012;109:69-75.

25. Liu CL, Peng LN, Chen YT, et al. Potentially inappropriate prescribing (IP) for elderly medical inpatients in Taiwan: a hospital-based study. Arch Gerontol Geriatr 2012;55:148-51.

26. Baldoni Ade O, Ayres LR, Martinez EZ, et al. Factors associated with potentially inappropriate medications use by the elderly according to Beers criteria 2003 and 2012. Int J Clin Pharm 2014;36:316-24.

27. Lai HY, Hwang SJ, Chen YC, et al. Prevalence of the prescribing of potentially inappropriate medications at ambulatory care visits by elderly patients covered by the Taiwanese National Health Insurance program. Clin Ther 2009;31:1859-70.

28. Chan DC, Hao YT, Wu SC. Characteristics of outpatient prescriptions for frail Taiwanese elders with long-term care needs. Pharmacoepidemiol Drug Saf 2009;18:327-34.

29. Kirschke M, Bohme J. [Impact of potentially inappropriate drug usage on health insurance business results]. Versicherungsmedizin 2014;66:142-5

30. Hovstadius B, Hovstadius K, Astrand B, et al. Increasing polypharmacy -an individual-based study of the Swedish population 2005-2008. BMC Clin Pharmacol 2010;10:16
31. Cashion W, McClellan W, Howard G, et al. Geographic region and racial variations in polypharmacy in the United States. Ann Epidemiol 2015;25:433-38 e1.

32. Stock S, Redaelli M, Simic D, et al. Risk factors for the prescription of potentially inappropriate medication (PIM) in the elderly: an analysis of sickness fund routine claims data from Germany. Wien Klin Wochenschr 2014;126:604-12.

33. Kim HA, Shin JY, Kim MH, et al. Prevalence and predictors of polypharmacy among Korean elderly. PLoS ONE 2014;9:e98043.

34. Galan Retamal C, Garrido Fernandez R, Fernandez Espinola S, et al [Prevalence of potentially inappropriate medication in hospitalized elderly patients by using explicit criteria]. Farm Hosp 2014;38:305-16

35. Reich O, Rosemann T, Rapold R, et al. Potentially inappropriate medication use in older patients in Swiss managed care plans: prevalence, determinants and association with hospitalization. PLOS ONE 2014;9:e105425.

36. Kaur S, Mitchell G, Vitetta L, et al. Interventions that can reduce inappropriate prescribing in the elderly: a systematic review. Drugs Aging 2009;26:1013-28.

37. Faustino CG, Passarelli MC, Jacob-Filho W. Potentially inappropriate medications among elderly Brazilian outpatients. Sao Paulo Med $J$ 2013;131:19-26.

38. Morin L, Fastbom J, Laroche ML, et al. Potentially inappropriate drug use in older people: a nationwide comparison of different explicit criteria for population-based estimates. Br J Clin Pharmacol 2015;80:315-24.

39. Hudhra K, Garcia-Caballos M, Jucja B, et al. Frequency of potentially inappropriate prescriptions in older people at discharge according to Beers and STOPP criteria. Int $J$ Clin Pharm 2014;36:596-603.

40. Jensen LD, Andersen O, Hallin M, et al. Potentially inappropriate medication related to weakness in older acute medical patients. Int $J$ Clin Pharm 2014;36:570-80.

41. Gray SL, LaCroix AZ, Hanlon JT, et al. Benzodiazepine use and physical disability in community-dwelling older adults. J Am Geriatr Soc 2006;54:224-30.

42. O'Sullivan DP, O'Mahony D, Parsons C, et al. A prevalence study of potentially inappropriate prescribing in Irish long-term care residents. Drugs Aging 2013;30:39-49.

43. Corsonello A, Onder G, Abbatecola AM, et al. Explicit criteria for potentially inappropriate medications to reduce the risk of adverse drug reactions in elderly people: from Beers to STOPP/START criteria. Drug Saf 2012;35(Suppl 1):21-8

44. Cool C, Cestac P, Laborde C, et al. Potentially inappropriate drug prescribing and associated factors in nursing homes. J Am Med Dir Assoc 2014;15:850.e1-9.

45. Marcum ZA, Hanlon JT. Commentary on the new American Geriatric Society Beers criteria for potentially inappropriate medication use in older adults. Am J Geriatr Pharmacother 2012;10:151-9. 